S. Caspi, M. Helm, and L.J. Laslett

Lawrence Berkeley Labozatory

University of California

Berkeley, California 94720

COMPUMAG

July $7-11,1991$

Sorrento, Italy

This report has been reproduced directly from the best available copy.

*This work was supported by the Director, Office of Energy Research, Office of High Energy and Nuclear Physics, Division of High Energy Physics, of the U.S. Department of Energy under Contraci No. DE-AC03-7óSF00098. 


\section{The Use of a Relaxation Method to Calculate the 3D Magnetic Field Contribution of an Iron Yoke*}

\author{
S. Caspi, M. Helm, and L.J. Laslett \\ Lawrence Berkelcy Labrratory \\ 1 Cyclotron Road, 3ldg. 46-161 \\ Berkeley, CA 94720
}

\begin{abstract}
A computational procedure has been developr! for calculating the threedimensional field produced by an axisymmetric iron yoke of high permeability in the presence of a system of conductors. The procedure is particulariy applicable to the end regions of multipole magnets of the sort used in particle accelerators. The field produced by the conductors is calculated using the BiotSavart law. We speak of the field contribution of the yoke as an "image field", although it is associated with a distinctly diffuse distribution of image currents or magntic momexts. At every point on the boundary of the yoke the total scalar potential is constant, so $v^{l}=-v^{d}$ where $i=$ image and $d=$ direct contribution from the conductors. If we describe both potentials as a series of "harmonic components" with respect to azimuthal dependence, then the nature of the boundary condition is such that a de-coupling of one harmonic component from another is preserved and therefore it is also true that $V^{i}(n)=-V^{d}(n)$ at the iron interface, where $n$ is a harmonic number. If we solve the appropriate individual differential equations for the scalar potential functions $v^{i}(n)$ throughout the iron-free region, with the proper applied boundary condition for the scalar potential of each harmonic number, we shall achieve upon summation the appropriate potential function to describe the field contribution of the surrounding high-permeability iron.
\end{abstract}

\section{INTRODUCTION}

Computations concerning the character and quality of magnetic ficlds produced by prescribed current distributions in the presence of ferromagnetic material are required in many technological applications, including such applications that employ

*This work was supported by the Director, Office of Energy Research, Office of High Energy and Nuclear Physics, Division of High Energy Physics, of the U.S. Department of Energy under Contract No. DE-AC03-76SF00098. superconducting material and must determine maximum values of magnetic ficlds in order to predict "short sample" performance of magnets. It is attractive to consider the use of finite-difference computational methods for the relaxation solution of such problems, but such techniques frequently have been judged inconvenient, if not inadequate, in threedimensional situations -. even if very simple approximations concerning the character of the ferromagnetic material can legitimately be assumed.

Our own contact with such issues has recently been in connection with the design of superconducting magnetic lens elements (dipoles, quadrupoles, etc.) for particle accelerators such as the $20 \times 20$ Tev Superconducting Super Collider (SSC). In this application, the length of the individual magnetic lenses is such that much useful information can be derived from two dimensional $(x, y$ or $r, \theta)$ computations in which one can specify in some detail the magnetic properties of the iron that surrounds the current winding and thus, through relaxation computations[1], achieve the requisite accuracy for describing the interior field that serves to guide and focus the particle beam. The effect of the end windings (and of the nearby iron in that region) also requires attention, however, since (i) the non-neglible contribution of the iron to field quality in this region must be estimated and (ii) the end configuration must be such as to reduce its indency to produce excessive field strength at the the superconducting windings. We wish to outline here some computational measures we have adopted to address such issues.

\section{ANALYSIS}

The magnetic field that arises "directly" from the current windings -- i.e. without inclusion of the contribution from induced magnetization in surrounding magnetic material .. of course can be computed at any point directly by application of the Biot-Savart law (possibly aided by simple numerical integration). At any desired longitudinal location (z) such field components (or, if desired, any associated magnetic-potential function) can be analyzed into Fourier components with respect to the azimuth angle $(\theta)$. Thus conventional "non-skewed" configurations may be expected to provide Fourier components (of index $\mathrm{n}$ ) for $\mathrm{B}_{\mathrm{r}}, \mathrm{B}_{\boldsymbol{\theta}}$, and $\mathrm{B}_{\mathrm{Z}}$ proportional respectively to $\sin n \theta, \cos n \theta$, and $\sin n \theta$. 
Such Fourier components of the "direct" field when evaluated in the region exterior to the current windings, in a location where surrounding magnetic material may be found, can usefully be employed to provide boundary conditions suitable for evaluation of the iron contribution to the total magnetic field -provided that (i) the iron interface is rotationally symmetric about the longitudinal axis (although possibly flared or interrupted with respect to the $z$ direction) and (ii) the iron may be regarded as characterized by a substantially infinite permeability. The individual harmonic components that the iron contributes to the total field may be described in the current-free region exterior to the current windings by functions $V_{n}(r, z)$, for which the scalar potential $V^{i}(r, \theta, z)$ for the iron contribution becomes the sum of such functions times $\sin n \theta$ while the individual functions $V_{n}(r, z)$ satisfy the differential equation

$$
\frac{1}{r} \frac{\partial}{\partial r}\left(r \frac{\partial V_{n}^{i}}{\partial r}\right)+\frac{\partial^{2} V_{n}^{i}}{\partial z^{2}}-\frac{n^{2}}{r^{2}} V_{n}^{i}=0
$$

in $r, z$ space, subject to the boundary condition that the individual Fourier components contributed by the iron have tangential components at the interface $(r=r b)$ that just cancel the corresponding "direct" tangential component of the field -- a condition that we specify by

$$
V_{n}^{i}\left(r_{b}, z\right)=-V_{n}^{d}\left(r_{b}, z\right)=\frac{r_{b}}{n} B_{\theta, n}^{d}\left(r_{b}, z\right)
$$

The completion of our problem thus requires only the solution of a (normally small) number of discrete two-dimensional partial differential equations, in $r, z$ space, and the combination of such solutions with the results calculated for the "direct" field.

An additional computational feature that may be applied to our two-dimensional solver, in correspondence to a feature we have introduced into POISSON, concerns the manner of imposing a surrounding boundary condition at the outermost, but finite, closed boundary of the two-dimensional mesh. The correct boundary condition in this case normally should be neither of the Dirichlet or Neumann type but should reflect the requirement that no "sources" (as currents or magnetized material) lie external to that boundary. In this case, we may construct in the outer regions of the mesh two, rather closely nested, boundary curves -- for example a pair of confocal cllipses with prolate cllipsoidal coordinates $\left(\xi, \eta_{\mathrm{i}}\right)$ and $\left(\xi, \eta_{0}\right)$. The character of the scalar potential function in this outer region may then be expressed in the present case $(n>0)$ as a sum of terms proportional to the product of associated Legendre functions
$P_{k}^{n}(\xi) Q_{k}^{n}(\eta)$ with $k \geq n$, in order to depict the falling off of an external potential with increasing distance from the axis. After the completion of one or more relaxation passes through the mesh, the coefficients of such a series development may be provisionally evaluated from the prevailing values of potential at mesh points along the inner boundary curve $\left(\eta=\eta_{i}\right)$ and the series then evaluated at points on the outer curve $\left(\eta=\eta_{0}\right)$ to provide revised potential values for resumption of the relaxation procedure.[2] This repetitive relaxation procedure has been found to converge satisfactorily and through use of associated

Legendre functions $P_{k}^{n}(\xi) Q_{k}^{n}(\eta)$, of order equal to the harmonic index $n$ and of degree $k \geq n$, could advantageously form a part of the modified relaxation program that is adopted to provide the scalar potential function $V_{n}^{i}$ in $r, z$ or $\eta, \xi$ space. It is a pleasure to acknowledge the support provided by Victor $O$. Brady, of this Laboratory, in adapting the program POISSON to solution of the partial differential equations for $V_{n}$. Further examination by a numerical analyst of these techniques and of possible modifications thereof could pruve to be of interest to workers in the field of advanced magnet design.

\section{A. Example - A Dipole Magnet}

We calculated the iron field contribution at the end region of a two layer superconducting dipole magnet (D19) currently under construction at our laboratory. The "end" windings, schematically shown in Figure 1 as line currents, were used to calculate the scalar potential harmonics at the iron boundary. Nine sets of potential (corresponding to $n=1,3,5 \ldots 17$, assuming dipole symmetry) have been applied as iron boundary values and used by the POISSON solver to form nine sets of solutions.

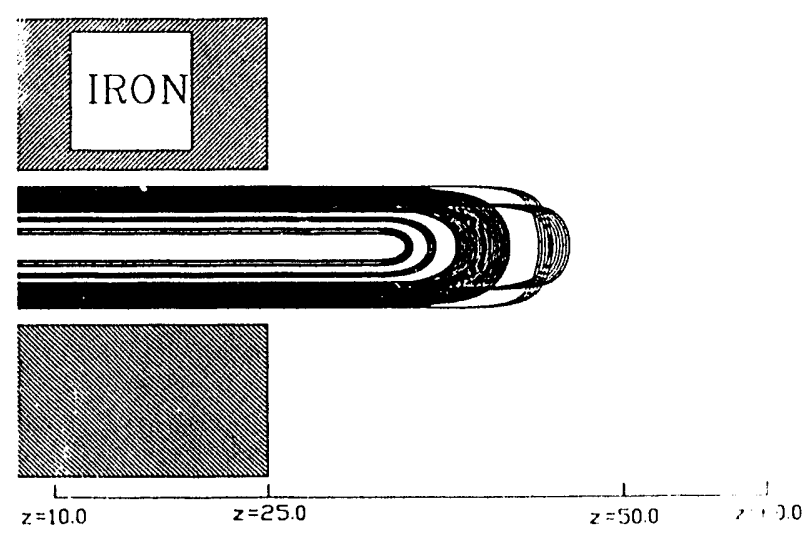

Fig. 1. Schematic top view of end region of double layer dipole Di?. 

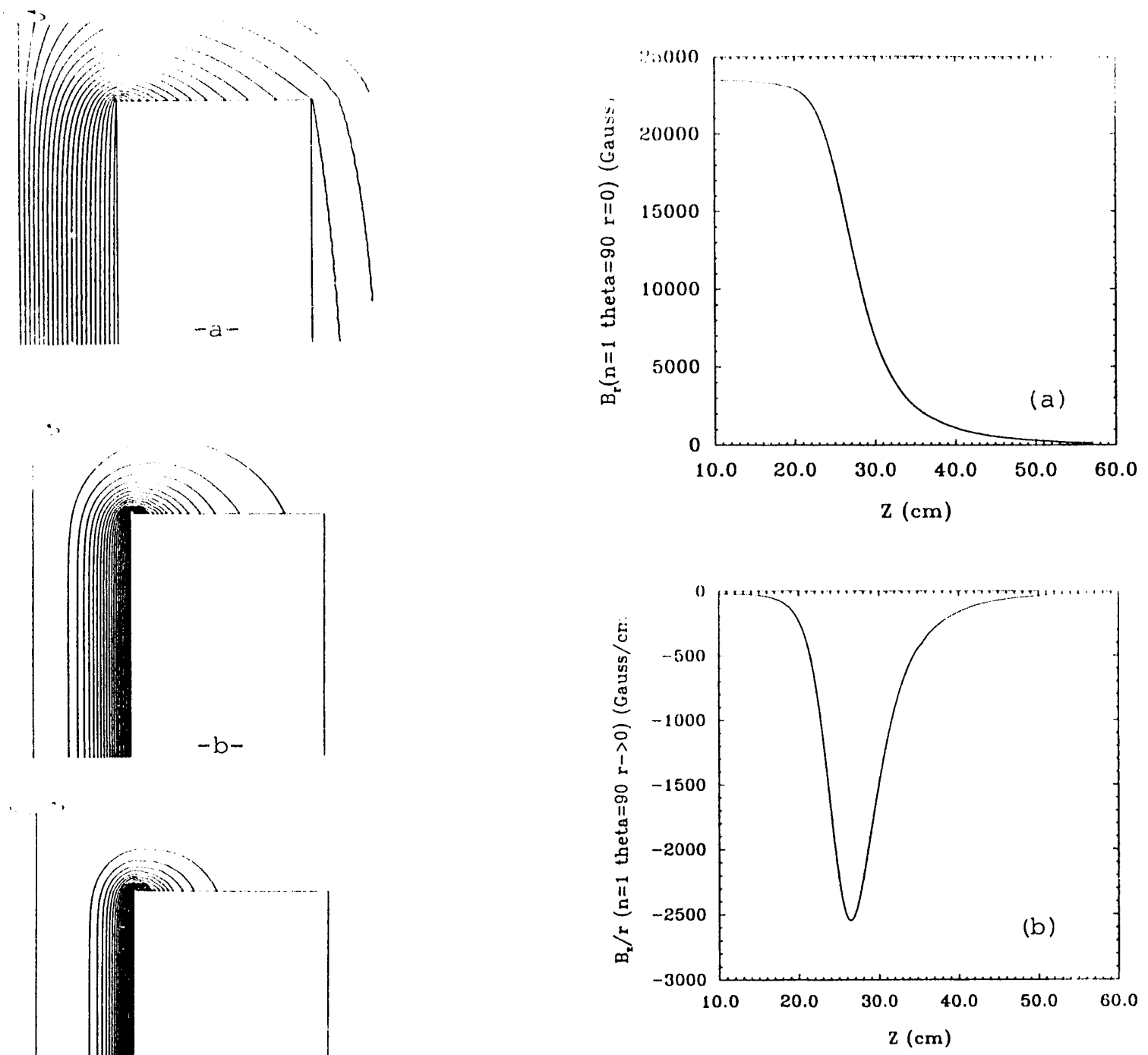

Fig. 3. Iron contribution to $B_{r}-a$, and to $B_{z}-b$, along the end region of D19.

Fig. 2. Equal potential lines for $a-n=1, b-n=3$, and $c-n=5$.

Plots of equal potential lines for 3 such sets are shown in Figure 2 for $n=1,3$, and 5 . We have used the fomulation belows to reconstruct the field components.

$$
\begin{aligned}
& B_{r}(r, \theta, z)=\sum_{n}-\frac{\partial V_{n}(r, z)}{\partial r} \sin (n \theta) \\
& B_{\theta}(r, \theta, z)=\sum_{n}-\frac{n}{r} V_{n}(r, z) \cos (n \theta) \\
& B_{z}(r, \theta, z)=\sum_{n}-\frac{\partial V_{n}(r, z)}{\partial z} \sin (n \theta)
\end{aligned}
$$

Finally, we plot in Figure $3 B_{r}(n=1, \theta=90, r=0)$ and $\frac{B_{Z}}{r}(n=1, \theta=90)$ in the limiting case $r \rightarrow 0$.

\section{REFERENCES}

[1] User's Guide for the POISSON/Superfish Group of Codes, Los Alamos Accelerator Code Group, LA-UK-87-115, January 1987.

[2] Analogous computational methods for various uses of Program POISSON are described for magnetostatic applications in LBL Report18798 (1984) and, more broadly, in LBL-24106 (1988). 

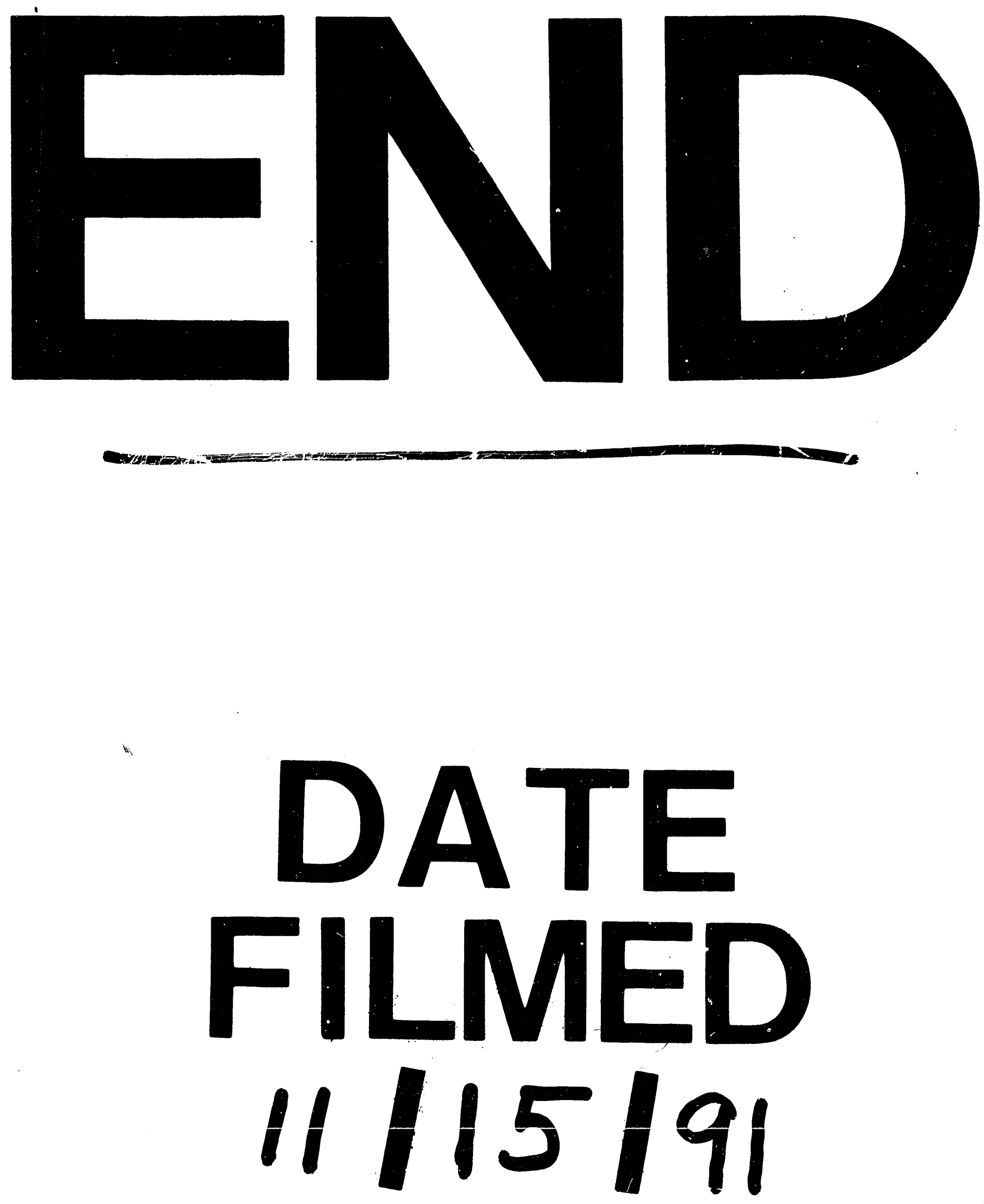
